\title{
CONSERVATIVE TREATMENT OF PANCREATIC NECROSIS WITH SUGGESTIVE SIGNS OF INFECTION
}

Enio Campos Amico, Leonardo Fernandes Canedo, Cibele Chuery Machado, Suzanna Gondim Faria, and Danilo Vendrame Vivas

Acute pancreatitis (AP) is a common disease in clinical practice. The treatment of the mild forms of AP is normally easy and effective. Nevertheless, the treatment of severe AP is generally associated with prolonged periods of intensive care hospitalization, high costs, and elevated morbidity and mortality rates. Fortunately, this last form occurs in only $10 \%$ to $20 \%$ of the patients. ${ }^{1}$

The development of the radiologic instruments, principally the CT scan, has been crucial to a better understanding of the disease. Hence, it is well-known that severe AP is related to the appearance of pancreatic and/or peripancreatic necrosis. ${ }^{2}$ Pancreatic necrosis represents a powerful stimulus to the systemic inflammatory response syndrome secondary to AP. This harmful amplified response may cause multiorgan failure, which is principally responsible for death in the first 2 weeks. Thereafter, the most dreaded and fatal complication is infection of the necrotic pancreas.

The necrotic pancreas may remain sterile, but almost $50 \%$ of the patients will eventually present a disseminated infection of the retroperitoneal tissue or a localized abscess. ${ }^{3}$ This fact is related to a significant increase of the morbidity and mortality rates, and for this reason, the consensus of AP management experts is to implement a surgical approach whenever an infected pancreatic necrosis is diagnosed. ${ }^{4,21}$

This paper presents a patient with necrotizing acute pancreatitis (NAP) associated with suggestive tomographic signs of infection who refused surgical management and was successfully treated by a conservative approach.

\section{CASE-REPORT}

A 63-year-old man, with a past medical history of car-

Surgery Department, Hospital Universitário da Faculdade de Medicina da Universidade do Oeste Paulista (UNOESTE) - Presidente Prudente/SP, Brazil.

Email: ecamic@uol.com.br diopathy and abdominal pain, was admitted to the emergency room complaining of diffuse abdominal pain associated with vomitting, abdominal distension, and fever that started 8 days before. Results of laboratory tests were as follows: hemoglobin $=12.3 \mathrm{~g} / \mathrm{dL}$, hematocrit $=37 \%$, leukocytes $=$ $12.320 \mathrm{p} / \mathrm{mm}^{3}$, amylase $=522 \mathrm{UI} / \mathrm{L}, \mathrm{BUN}=44 \mathrm{mg} / \mathrm{dL}, \mathrm{Cr}$ $=1.2 \mathrm{mg} / \mathrm{dL}$. Abdominal ultrasound revealed that the head of the pancreas was increased in size and heterogenic with the presence of peripancreatic liquid, which was suggestive of AP. Conservative treatment consisting of withholding oral intake, fluid replacement, and analgesia was initiated. A CT scan using intravenous contrast media was obtained, and total parenteral nutrition was also started because of the persistence of significant abdominal pain and high blood levels of amylase. The CT scan showed areas of necrosis at the pancreatic head and body, associated with the presence of gas in the retrogastric and anterior pararenal spaces (Figure 1). Surgery was indicated, and antibiotic therapy was simultaneously initiated using ciprofloxacin (1g/day) and metronidazole ( $1.5 \mathrm{~g} /$ day). The patient refused surgery and was transferred to another hospital, where he was evaluated by another medical team. Conservative treatment with total parenteral nutrition and antibiotic therapy was maintained. After 3 days, the patient became afebrile; oral feeding was reintroduced after 6 days, and he was discharged with no symptoms 13 days after the beginning of treatment. No clinical sign of complications was identified over a 4 month follow-up period, at which time, an ultrasound test showed that the abdominal liquid had been reabsorbed.

\section{DISCUSSION}

The treatment of NAP has changed considerably over the past 20 years; mortality rates dropped from $50 \%-70 \%$ in the seventies to $20 \%,{ }^{5}$ nowadays. Nevertheless, infection is still a very prevalent complication in these patients, 


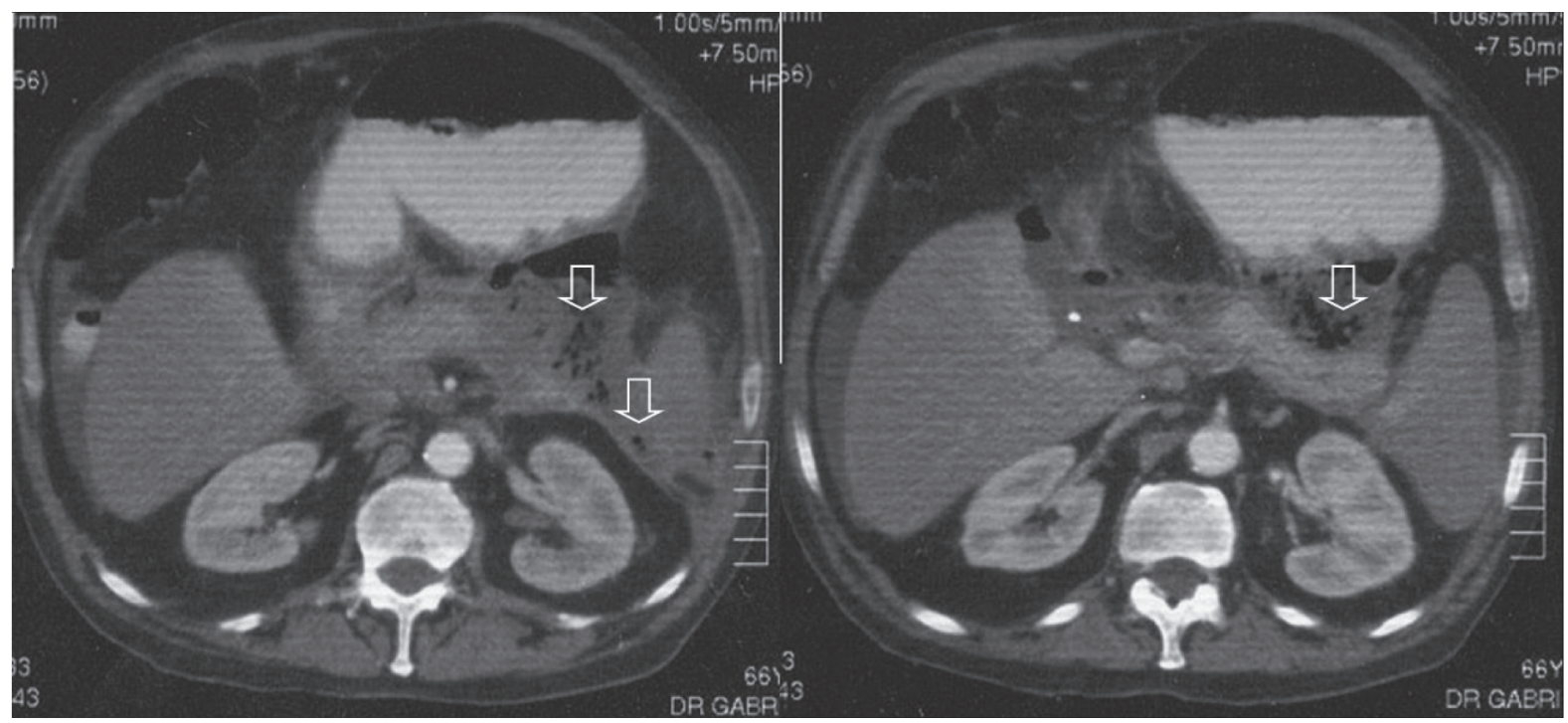

Figure 1 - Abdominal CT scan using intravenous and oral contrast media demonstrating small necrotic areas in the head and body of the pancreas, infiltration of peripancreatic fat, and presence of gas in the retrogastric and pararenal spaces (arrows)

being responsible for $80 \%$ of the deaths. According to the guidelines established by the International Association of Pancreatology at the Symposium of Heidelberg ${ }^{6}$, once the diagnosis of infection in pancreatic necrosis has been established, a surgical necrosectomy should be always carried out, in view of the high mortality rates associated with the conservative approach. Although the CT scan is the "gold standard" for the diagnosis of pancreatic necrosis, the presence of an infectious complication, which is generally difficult to establish, may drastically change the management. ${ }^{7}$ For this reason, patients under suspicion of an infected necrotic pancreas have undergone percutaneous aspiration of the necrotic tissue because of its high accuracy and superiority over clinical observations, laboratory tests, or radiological data. ${ }^{8}$

The scenario of an anaerobic infection represents a special situation. These germs do not have the cytochrome system involved in oxygen metabolism. Thus, their growth and metabolism are achieved through the energy released during fermentation reactions; $\mathrm{CO}_{2}$ and $\mathrm{H}_{2}$ are also produced, forming air bubbles in the infected tissues. Consequently, it is accepted that the identification of extraluminal air in the necrotic pancreas or peripancreatic liquid corresponds to the only pathognomonic tomographic sign of infection. ${ }^{9}$

Recent series concerning the management of patients with NAP have described the presence of extraluminal gas as an indicative of necrosectomy, avoiding a confirmatory percutaneous aspiration. Yousaf et al., in a review paper, proposed an algorithm for the management of NAP, in which the identification of gas by CT scan has the same meaning as a positive culture obtained from a percutaneous aspiration and indicative of a bad clinical evolution, requiring surgery. ${ }^{7}$ The role of the anaerobic germs in severe NAP has probably been underestimated, since only $1 \%$ to $3 \%$ of the cultures of the aspirates or surgical specimens are positive, while the tomographic sign of extraluminal air is found in $3.7 \%$ to $12.5 \%$ of the patients..$^{5,10,11,12,13}$ Whenever present, the anaerobic infection of the pancreatic necrosis is highly lethal.

The clinical evolution of the reported case was unusual. Two hypotheses may be discussed: the existence of another source for the extraluminal gas or a rare benign evolution of a selected group of patients affected by anaerobic infection of pancreatic necrosis. Another possibility, albeit remote, is a false positive of the tomographic sign of extraluminal gas for anaerobic infection, since bacteriologic confirmation, the "gold standard", was not achieved

Possible causes for the extraluminal gas could be an enteric fistula or duodenal and colonic rupture, even though there was no extravasation of the oral contrast media on the CT scan. Furthermore, these complications normally undergo an adverse evolution that should also be treated by surgical means. Since the patient did not undergo diagnostic aspiration, the possibility of an extraluminal source of gas may not be excluded.

The second hypothesis is that perhaps, for a selected group of patients, surgical management may be avoided. This approach would have the potential benefit of reducing some of the complications related to the procedure, which is usually repetitive, as well as the length of hospitalization and, consequently, the costs. ${ }^{14}$

Percutaneous treatment has also been described recently by some authors as a less invasive alternative for the management of infected NAP. Freeny et al. were successful in 
$16(47 \%)$ of 34 patients with infected NAP using percutaneous drainage associated with daily irrigation. ${ }^{15}$ Better results were achieved by Carter et al. ${ }^{14}$ who successfully treated with percutaneous drainage and endoscopic necrosectomy 8 out of 10 patients with confirmatory positive aspiration culture of the necrotic tissue; these patients would otherwise have undergone open necrosectomy. Regarding complications, one patient had a laparotomy because of a splenic vessel hemorrhage. These reports present alternatives to open surgical removal of infected pancreatic necrosis as an important step for the management of these patients. None of this was not done in the case presented in this report.

The treatment of probably infected necrosis mainly by antibiotic therapy, as presented in this paper, is reported by a few authors who studied limited numbers of patients. ${ }^{16}$ Baril et al. ${ }^{17}$ described six patients with infected peripancreatic liquid treated only by antibiotic therapy, with a mortality rate of $33.3 \%$. Rünzi et al., ${ }^{18}$ treating six patients in the same manner avoided surgery in $53.8 \%$ of the cases with a mortality rate of only $8 \%$, a result similar or even better than that following an invasive approach. Recently, Whitelaw et al. reported that $12.3 \%$ of the 65 patients with infected pancreatic necrosis who were treated conservatively avoided percutaneous drainage with satisfactory results. ${ }^{19}$
The best scientific evidence in support of the nonsurgical management of infected NAP was presented by Cancino et $\mathrm{al}^{20}$, who prospectively studied 22 nonselected patients diagnosed by aspiration (15 patients) or by the presence of extraluminal gas (4 patients), without manifest signs of organ insufficiency. Ciprofloxacine and a third or a fourth generation cephalosporin, associated with antianaerobic drugs, were used for approximately 20 days. Good clinical response was unequivocal in 19 patients, most of whom became afebrile within the first 48 hours of treatment. Three patients underwent a limited surgical approach without morbidity and early hospital discharge. No deaths occurred in this series.

We conclude that the conservative approach adopted for the patient reported herein, permitted the effective treatment of the infected pancreatic necrosis without the high costs related to the recommended surgical management. In spite of the uncertainty regarding the factors responsible for the favorable response to antibiotic monotherapy and the weak evidentiary power of case reports, this paper suggests that, for a selected group of patients, mainly those with a good clinical condition in which a fast response to antibiotic therapy is achieved, the conservative approach may avoid the need for necrosectomy.

\section{REFERENCES}

1. Beger HG, Rau B, Isenmann R. Natural history of necrotizing pancreatitis. Pancreatology. 2003;3:93-101.

2. Bradley III EL. A clinically based classification system for acute pancreatitis. Arch Surg. 1993;128:586-90.

3. Ratschko M, Fenner T, Lankisch PG. The role of antibiotic prophylaxis in the treatment of acute pancretitis. Gastroenterol Clin North America. 1999;28:641-59.

4. Wyncoll DL. The management of severe acute necrotizing pancreatitis: an evidence-based review of the literature. Intensive Care Med. 1999;25:146-56.

5. Branum G, Galloway J, Hirchowitz W, Fendley M, Hunter J. Pancreatic necrosis. Results of necrosectomy, packing, and ultimate closure over drains. Ann Surg. 1998;227:870-7.

6. Uhl W, Warshaw A, Imrie C, Bassi C, McKay CJ, Lankisch PG et. al. IAP guidelines for the surgical management of acute pancreatitis. Pancreatology. 2002;2:565-73.

7. Yousaf M, McCallion K, Diamond T. Management of severe acute pancreatitis. Br J Surg. 2003;90:407-20.

8. Gerzof SG, Banks PA, Robbins AH, Johnson WC, Spechler SJ, Wetzner SM, et al. Early diagnosis of pancreatic infection by computed tomography-guided aspiration. Gastroenterology. 1987;93:1315-20.
9. Duininck TM, Libsch KD, Ueno T, Sarr MG. Infected pancreatic necrosis. Problems in General Surgery. 2002;19:72-80.

10. Ashley SW, Perez A, Pierce EA, Broks DC, Moore Jr FD, Whang EE, et al. Necrotizing pancreatitis. Contemporary analysis of 99 consecutive cases. Ann Surg. 2001;234:572-80.

11. Büchler MW, Gloor B, Müller CA, Friess H, Seiler CA, Uhl W. Acute necrotizing pancreatitis: treatment strategy according to the status of infection. Ann Surg. 2000;232:619-26.

12. Büchler P, Reber HA. Surgical approach in patients with acute pancreatitis. Is infected or sterile necrosis an indication-in whom should this be done, when, and why? Gastroenterol Clin N Am. 1999;28:66171.

13. Del Castillo CF, Rattner DW, Makary MA, Mostafavi A, McGrath D, Warshaw AL. Débridement and closed packing for the treatment of necrotizing pancreatitis. Ann Surg. 1998;228:676-84.

14. Carter CR, McKay CJ, Imrie CW. Percutaneous necrosectomy and sinus tract endoscopy in the management of infected pancreatic necrosis: an initial experience. Ann Surg. 2000;232:175-80.

15. Freeny PC, Hauptmann E, Althaus SJ, Traverso LW, Sinanan M. Percutaneous CT-guided catheter drainage of infected acute necrotizing pancreatitis: techniques and results. AJR. 1998;170:969-75. 
16. Faintuch J, Meniconi MT, Speranzini MB, Pinotti HW, Smolentsov H. Clinical regression of infected pancreatic necrosis. Case report. Int $\mathbf{J}$ Pancreatol. 1991;8:379-86.

17. Baril NB, Ralls PW, Wren SM, Selby RR, Randin R, Parekh D et al. Does an infected peripancreatic fluid collection or abscess mandate operation? Ann Surg. 2000;231:361-7.

18. Rünzi M, Layer P. Nonsurgical management of acute pancreatitis. Use of antibiotics. Surg Clin North America. 1999;79:759-65.
19. Whitelaw D, Shankar A, Aggrwal R, Russel RCG, Kurzawinski TR. Management of acute severe pancreatitis without necrosectomy. Br J Surg. 2004;91:516-517.

20. Cancino NA, Torres RO, Schwaner CJ. Tratamiento antibiótico de la pancreatitis aguda necrotizante infectada. Rev Chil Cir. 2002;54:600-4.

21. Pancreatic Disease Group, Chinese Society of Gastroenterology \& Chinese Medical Association. Consensus on the diagnosis and treatment of acute pancreatitis. Chinese Journal of Digestive Diseases. 2005;6:47-51. 